\title{
13 Projektpartner/innen
}

Dieses Kapitel wurde bei Erstveröffentlichung ohne die korrekte Creative Commons Lizenz veröffentlicht. Die korrekte Lizenz finden Sie am Ende des Kapitels.

Ein Erratum zu diesem Kapitel ist verfügbar unter DOI 10.1007/978-3-658-12533-2_14

(C) SINUS Markt- und Sozialforschung GmbH 2016

M. Calmbach et al., Wie ticken Jugendliche 2016?,

DOI 10.1007/978-3-658-12533-2_13 


\section{aff $\square$}

Arbeitsstelle für Jugendseelsorge der Deutschen Bischofskonferenz

Die Arbeitsstelle für Jugendseelsorge der Deutschen Bischofskonferenz (afj) ist als Fachstelle für Jugendfragen in die Arbeit der Jugendkommission und des Sekretariats der Deutschen Bischofskonferenz eingebunden.

Sie gliedert sich in vier Fachreferate:

$>\quad J u g e n d p a s t o r a l e ~ B i l d u n g$

(7) Glaubensbildung

( Ministrantenpastoral und liturgische/kulturelle Bildung

(7) Politische Bildung.

Als afj sind wir gemeinsam mit dem Bund der Deutschen Katholischen Jugend (BDKJ) Träger des Jugendhauses Düsseldorf e. V., der Bundeszentrale für katholische Jugendarbeit.

Wir fördern, koordinieren und entwickeln die Jugendpastoral im nationalen und internationalen Bereich durch:

( die Beobachtung und Begleitung aktueller Prozesse in der Jugendpastoral

( die Förderung des Austausches zwischen den verschiedenen Trägern kirchlicher Jugendarbeit und die Vermittlung von Anregungen für die Praxis 
( die Durchführung der Jahreskonferenz Jugendseelsorge zur Anregung des überdiözesanen Austauschs und der kollegialen Beratung von Akteuren der Jugendpastoral

( die Zusammenarbeit mit und die Zuarbeit für die Jugendkommission der Deutschen Bischofskonferenz

( die Durchführung von Fachtagungen und Fortbildungen, Publikationen in Zeitschriften und Büchern sowie die Konzipierung von Vorträgen und Forschungsprojekten

( die Koordination der nationalen Plattform des „Katholischen Netzwerkes Jugend in Europa" - Rete Juventutis, dessen Gründungsmitglied wir sind

( die Unterstützung und Förderung der Begegnung katholischer Jugend auf internationaler Ebene

(>) die nationale Koordination der Weltjugendtage.

Unsere Arbeit bringen wir auf die Kurzformel:

Vernetzen - Unterstützen - Vordenken

Vernetzen - Wir pflegen den institutionellen und persönlichen Austausch mit allen wichtigen Akteuren und Verantwortlichen der Jugendpastoral in Deutschland sowie auf europäischer Ebene. Wir bringen Anliegen zusammen und vermitteln qualifizierte Kontakte.

Unterstützen - Den haupt- und ehrenamtlichen Akteuren der Jugendpastoral stellen wir aktuelle Ergebnisse aus Forschung und Praxis zur Verfügung. Mit Materialien und Arbeitshilfen bieten wir unterschiedlichen Zielgruppen konkrete Unterstützung für ihre Arbeit.

Vordenken - Mit unserem Überblick über Praxis und Wissenschaft entwickeln wir aktuelle Konzepte, bringen neueste Ergebnisse in die Diskussion ein und verschaffen wichtigen Anliegen Gehör. So geben wir Impulse für die Reflexion und Entwicklung der Jugendpastoral in Deutschland.

www.afj.de 
Der Bund der Deutschen Katholischen Jugend (BDKJ) ist der Dachverband von 17 katholischen Jugendverbänden und -organisationen. Seine wichtigste Aufgabe besteht in der Interessenvertretung seiner Mitglieder in Politik, Kirche und Gesellschaft.

Der BDKJ vertritt rund 660.000 Kinder, Jugendliche und junge Erwachsene im Alter zwischen 7 und 28 Jahren. Der BDKJ ist organisiert in Kommunen, Kreisen, Bundesländern und im Bundesgebiet sowie in kirchlichen Territorien wie Dekanaten, Regionen und in 26 deutschen Bistümern. Er hat seine Wurzeln in der Jugendbewegung, die ab dem Ende des 19. Jahrhunderts zur Gründung zahlreicher, auch katholischer Jugendverbände führte. Nach dem Verbot der konfessionellen Jugendverbandsarbeit in der nationalsozialistischen Diktatur gab sich die katholische Jugend mit der Gründung des BDKJ 1947 eine neue Ordnung.

Der BDKJ will Mädchen und Jungen zu kritischem Urteil und eigenständigem Handeln aus christlicher Verantwortung befähigen und anregen. Er wirkt bei der Entwicklung von Kirche, Gesellschaft, Staat und internationalen Beziehungen mit, um unsere Gesellschaft und Kirche zukunftsfähig und die Weltgemeinschaft gerechter und solidarischer zu gestalten. Dieses Engagement wird getragen von Kindern, Jugendlichen und jungen Erwachsenen, die sich auf den verschiedenen Ebenen des BDKJ freiwillig und ehrenamtlich engagieren. Demokratische Organisation, Ermutigung zu Selbstorganisation und Partizipation und ein konsequenter Lebensweltbezug sind wichtige Säulen der katholischen Jugendverbandsarbeit. 
Fairer Handel, Klimagerechtigkeit und kritischer Konsum, jugendgerechte Politik, Mädchen- und Frauenpolitik, Jugendsozialarbeit, Freiwilligendienste und aktuelle Herausforderungen wie etwa der Einsatz für Geflüchtete sind die Themenfelder, die die Arbeit des BDKJ maßgeblich prägen und mit denen er sich für Frieden, Gerechtigkeit und Bewahrung der Schöpfung einsetzt. Das Engagement des BDKJ ist nicht denkbar ohne seine eindeutige Verortung als katholische Organisation, es wird vom christlichen Glauben getragen und motiviert. Der BDKJ ist eine gesellschaftliche Kraft in der Kirche wie auch eine kirchliche Kraft in der Gesellschaft. Er versteht sich als "katholisch - politisch - aktiv“ und bietet als Dachverband Unterstützung für die in ihm organisierten Kinder, Jugendlichen und jungen Erwachsenen, die sich auf vielfältige Weise und in unterschiedlichsten Zusammenhängen engagieren, Gemeinschaft erleben und ihren Glauben feiern.

www.bdkj.de 


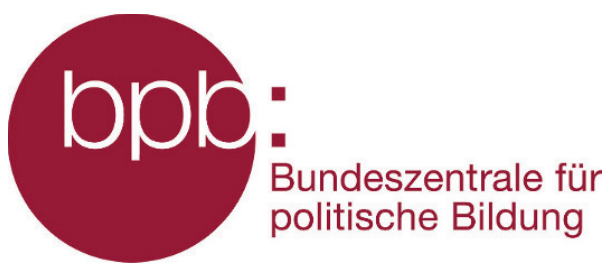

Demokratie stärken - Zivilgesellschaft fördern

Im Zentrum der Arbeit der Bundeszentrale für politische Bildung/bpb steht die Förderung des Bewusstseins für Demokratie und politische Partizipation. Aktuelle und historische Themen greift sie mit Veranstaltungen, Printprodukten, audiovisuellen Produkten und durch Online-Angebote auf. Veranstaltungsformate der bpb sind Tagungen, Kongresse, Festivals, Messen, Ausstellungen, Studienreisen, Wettbewerbe, Kinoseminare und Kulturveranstaltungen sowie Events und Journalistenweiterbildungen. Das breit gefächerte Bildungsangebot der bpb soll Bürgerinnen und Bürger motivieren und befähigen, sich kritisch mit politischen und gesellschaftlichen Fragen auseinander zu setzen und aktiv am politischen Leben teilzunehmen.

Seit 1952 engagiert sich die bpb für die Stärkung der Demokratie und der Zivilgesellschaft - zunächst in der alten Bundesrepublik und seit 1989 in ganz Deutschland. Die unterschiedlichen Bildungsangebote vermitteln Einblicke in die historischen und gesellschaftlichen Zusammenhänge politischer, kultureller, sozialer sowie wirtschaftlicher Prozesse. Ihre Aufgabe erfüllt die bpb in eigener gesellschaftspolitischer, pädagogischer und publizistischer Verantwortung. Sie ist überparteilich und wissenschaftlich ausgewogen. Als eine Institution der staatlich verfassten politischen Bildung unterstützt sie bundesweit Einrichtungen, Nicht-Regierungsorganisationen, Stiftungen und Vereine, die politische Bildung anbieten. 
Die bpb hält besondere Angebote für Lehrerinnen, Lehrer und Personen in der Bildungs- und Jugendarbeit bereit. Jugendliche und junge Erwachsene spricht sie mit altersgemäßen Themen und Medien direkt an. Sie erarbeitet spezielle Medienpakete und Fortbildungen für junge Erwachsene in Sportvereinen, bei Bundeswehr oder Polizei. Die bpb macht sich moderne Kommunikationsmethoden zu eigen und verfolgt einen crossmedialen Ansatz. Sie stellt sich den Anforderungen nach schneller und fundierter Information: Mit ihren Bildungsangeboten und speziellen Online-Produkten greift sie aktuelle gesellschaftliche sowie politische Ereignisse und Debatten auf. 


\section{deutsche kinder- und jugendstiftung}

Die Deutsche Kinder- und Jugendstiftung (DKJS) setzt sich dafür ein, allen jungen Menschen gesellschaftliche Teilhabe und Bildungserfolg zu ermöglichen. Besonders Kinder und Jugendliche, die in Risikolagen aufwachsen, stehen dabei im Fokus. Die DKJS vernetzt Akteure aus Staat, Wirtschaft, Wissenschaft, Praxis und Zivilgesellschaft und entwickelt mit ihnen praktische Antworten auf aktuelle Herausforderungen im Bildungssystem. In ihrer Arbeit lenkt sie den Blick auf die Stärken, nicht auf die Defizite von Kindern und Jugendlichen.

In diesen Handlungsfeldern ist die DKJS mit ihrer Expertise aktiv:

(7) Frühe Bildung

(7) Schulerfolg \& Ganztagsschule

(>) Bildungslandschaften

(7) Jugend \& Zukunft

Die Themen Leben und Lernen im digitalen Alltag und junge Geflüchtete spielen aktuell eine besonders wichtige Rolle.

Als operative Stiftung konnte die DKJS seit 1994 mehr als 500 Programme und Projekte für Kinder und Jugendliche sowie ihre erwachsenen Begleiter realisieren. Dabei setzt sie als unabhängige, überkonfessionelle und parteipolitisch neutrale Initiative auf wirkungsvolle Kooperationen mit Partnern der öffentlichen Hand und Zivilgesellschaft. 
Im Rahmen der SINUS-Studie interessiert die DKJS, welches Bild Jugendliche von der Digitalisierung und deren Auswirkungen auf ihren Alltag und ihre Zukunft haben; wie bewusst und kompetent sie mit digitalen Medien umgehen; welche Unterschiede je nach Lebenswelt es gibt und was sie sich von den Erwachsenen, insbesondere in der Schule, als Unterstützung wünschen.

Noch wenig untersucht ist die Frage, wie digitale Medien direkt das Lernen und Denken von Kindern und Jugendlichen prägen. Dominiert die reine Nutzung von Software in einer eher passiven Rolle? Mehr Lernpotential und Lebensweltorientierung entsteht, wenn Kinder selbst aktiv werden und erleben, wie sie Technik auch lenken und kontrollieren können. Hier macht die DKJS bereits sehr gute Erfahrungen im Camp-Format KIDScraft und wünscht sich, praxisrelevante Forschung, um in ihrer Programmarbeit darauf aufbauen zu können.

Mehr Informationen: www.dkjs.de 


\section{VDV Akademie}

Die VDV-Akademie - Bildung in Bewegung

Seit 2001 ist die VDV-Akademie in der beruflichen Weiterbildung unterwegs. Mit Lehrgängen, Tagungen und Kongressen erreicht sie die Menschen in den Verkehrsunternehmen, die mit ihren Dienstleistungen für Mobilität in Deutschland sorgen. Als Bildungstochter des Verbandes Deutscher Verkehrsunternehmen (VDV) hat sie sich mit jährlich über 2.000 Teilnehmern zu einem der wichtigsten Qualifizierungs- und Tagungsanbieter für die Bus- und Bahnbranchen entwickelt.

Die VDV-Akademie bereitet ihre Bildungsteilnehmer auf Abschlüsse und Prüfungen für staatlich anerkannte und branchenbezogene Berufsbilder vor.

Wie und wo werden wir in Zukunft lernen? - Im Kontext von Digitalisierung, Vernetzung und Nachhaltigkeit treibt diese Frage auch die Akademie als Bildungsanbieter um. Strategisch geht es um die Weiterentwicklung des „Blended-Learning-Modells", also einer guten Mischung aus Präsenzlernen und Online-Lernen. Operativ sieht sich die VDV-Akademie vor die Herausforderung gestellt, mit ihrem digitalen Ansatz den Teilnehmenden an Qualifizierungsmaßnahmen die Möglichkeit zu bieten, zeitlich und örtlich unabhängig, anhand von digitalen Lehrunterlagen, speziell aufbereiteten Trainingseinheiten, Videoclips, Webinaren und präsenzvorbereitenden Aufgabenstellungen zu lernen. Ziel ist es, die "Lerntemperaturen" in den Qualifizierungsmaßnahmen und damit die Lernerfolge der Teilnehmer zu erhöhen. 
Die VDV-Akademie tritt auch als Herausgeberin von Lehrbüchern für verkehrsspezifische Berufsbilder auf, erstellt Gutachten und publiziert fachbezogene, praxisnahe Leitfäden und Werkzeugkoffer für die Branchenunternehmen.

In Workshops und "Denkfabriken" beschäftigt sie sich mit den Erwartungshaltungen von Jugendlichen an die zukünftige Mobilität und diskutiert, wie junge Menschen als Fahrgäste und Mitarbeitende für Verkehrsunternehmen gewonnen werden können.

Michael Weber-Wernz

Geschäftsführer 


\section{SInUS}

Partner von INTEGRAL Wien

SINUS ist ein unabhängiges, inhabergeführtes Institut für psychologische und sozialwissenschaftliche Forschung und Beratung

Verstehen, was Menschen bewegt: Mit ethnologischer Neugier, Empathie, Respekt, wissenschaftlicher Verlässlichkeit und nicht zuletzt über 30 Jahren Erfahrung erforscht SINUS in vielen Ländern die Alltagswirklichkeit der Menschen, den gesellschaftlichen Wandel und seine Bedeutung für Unternehmen, Institutionen und Regierungen.

(7) SINUS analysiert Motive und Verfassungen der Menschen

(7) SINUS beschreibt Zielgruppen

(7) SINUS identifiziert soziokulturelle Trends \& Treiber

(>) SINUS führt mit Szenarien in die Zukunft

( SINUS setzt Forschungsergebnisse in strategische Beratung um

SINUS unterhält eine Vielzahl nationaler und internationaler Forschungsund Beratungskooperationen und hat weltweit ein Netzwerk von Experten aus den unterschiedlichsten Disziplinen aufgebaut: universitäre und privatwirtschaftliche Forschungsinstitute, führende Agenturen und Berater, spezialisierte Forschungsinstitute, und namhafte Designexperten kooperieren seit Jahren mit SINUS. 
Das bekannteste Produkt von SINUS sind die Sinus-Milieus. Die Sinus-Milieus gruppieren Menschen, die sich in ihrer Lebensauffassung und Lebensweise ähneln. Grundlegende Wertorientierungen gehen dabei ebenso in die Analyse ein wie Alltagseinstellungen zu Arbeit, Familie, Freizeit, Geld und Konsum. Sie rücken also den Menschen und das gesamte Bezugssystem seiner Lebenswelt ganzheitlich ins Blickfeld. Damit bieten sie mehr Informationen und bessere Entscheidungshilfen als herkömmliche Zielgruppenansätze. Die Einsatzgebiete der Sinus-Milieus sind dabei breit gefächert. Neben Anwendungen in Politik, Kirche, Publizistik oder Pädagogik gibt es in der klassischen Marketingforschung kaum eine Marktkategorie, für welche die Sinus-Milieus noch nicht eingesetzt wurden.

www.sinus-institut.de 


\section{SInUS:akademie ${ }^{\circledR}$}

Qualifizierung, Beratung, Innovation

Die SINUS:akademie ist das Weiterbildungs- und Beratungsangebot des SINUS-Instituts. Ihr Anliegen ist es, die Erkenntnisse der SINUS Markt und Sozialforschung für Unternehmen und Organisationen nutzbar zu machen.

Mit einem umfassenden Angebot an Vortragsformaten wie Keynotes, Impuls- und Fachvorträgen sowie Vertiefungsformaten wie Workshops, Weiterbildungen und Beratungsleistungen wird das Forschungs- und Beratungs-Know-how des SINUS-Instituts und seiner Consultancy Partner praxisnah und kundenorientiert ausgebaut.

Die Referentinnen und Referenten aus dem Team der SINUS:akademie bringen neben dem exklusiven SINUS-Wissen eine große Breite an fachlichen Hintergründen aus ihrer Berufspraxis ein, von der pädagogischen und politischen Arbeit über Betriebswirtschaft und Marketing bis hin zu Coaching und Supervision. Mit rund 500 Veranstaltungen für mehr als 400 Kundinnen und Kunden seit der Studie "Wie ticken Jugendliche 2012?" schöpft die SINUS:akademie aus einem breiten Erfahrungsschatz und fundiertem Detailwissen zu den Themen Jugend und Zielgruppen der Zukunft. 
Die SINUS:akademie bietet inhaltlich und methodisch maßgeschneiderte Lösungen für Unternehmen, Verbände, soziale und kommunale Einrichtungen, politische Organisationen und alle, die ihre Arbeit auf der Basis aktueller Markt- und Sozialforschung erfolgreich weiterentwickeln wollen.

\section{Alle Informationen zum Veranstaltungsangebot der SINUS:akademie zur SINUS-Jugendstudie finden Sie unter www.wie-ticken-jugendliche.de}

Open Access Dieses Kapitel wird unter der Creative Commons Namensnennung - Nicht kommerziell 2.5 International Lizenz (http://creativecommons.org/licenses/by-nc/2.5/deed.de) veröffentlicht, welche für nicht kommerzielle Zwecke die Nutzung, Verbreitung und Wiedergabe in jeglichem Medium und Format erlaubt, sofern Sie den/die ursprünglichen Autor(en), den Titel des Werks und die Quelle ordnungsgemäß nennen, einen Link zur Creative Commons Lizenz beifügen und im Falle einer Abwandlung durch einen entsprechenden Hinweis deutlich erkennbar machen, dass Änderungen vorgenommen wurden.

Die in diesem Kapitel enthaltenen Bilder und sonstiges Drittmaterial unterliegen ebenfalls der genannten Creative Commons Lizenz, sofern sich aus der Abbildungslegende nichts anderes ergibt. Sofern das betreffende Material nicht unter der genannten Creative Commons Lizenz steht und die betreffende Handlung nicht nach gesetzlichen Vorschriften erlaubt ist, ist auch für die oben aufgeführten nicht-kommerziellen Weiterverwendungen des Materials die Einwilligung des jeweiligen Rechteinhabers einzuholen. 